\title{
Early Growth Response 3 regulates genes of inflammation and directly activates IL6 and IL8 expression in prostate cancer
}

\author{
V T Baron ${ }^{1}, \mathrm{R} \mathrm{Pio}^{2}, \mathrm{Z} \mathrm{Jia}^{2,3,4}$ and D Mercola*,2 \\ ${ }^{1}$ Vaccine Research Institute of San Diego, San Diego, CA 92109, USA and ${ }^{2}$ Department of Pathology and Laboratory Medicine, \\ University of California, Irvine, Irvine, CA 92067-4800, USA
}

Background: Transcription factor EGR3 (Early Growth Response 3) is a little-studied member of the EGR family that is highly expressed in human prostate tumours compared with normal tissue. Its function in prostate cancer, however, is unknown.

Methods: Stable shRNA silencing was achieved in naturally overexpressing prostate cancer cells, followed by Affymetrix expression analysis. Fold changes of $\geqslant 2$ and $\leqslant-2$ were considered valid and $t$-tests $P$-values of $\leqslant 0.01$ were considered statistically significant. Potential EGR3 target genes were validated by real-time qPCR, chromatin immunoprecipitation, and gainof-function experiments. Promoter analysis confirmed the presence of consensus binding sites in the promoters of target genes.

Results: Early Growth Response 3 regulates the expression of $\sim 330$ genes, 35\% of which are involved in immune responses and inflammatory processes, and $15 \%$ crosstalk with the NF- $\kappa$ B signalling pathway. In particular, EGR3 induces the expression of over 50 secreted cytokines, growth factors, and matrix remodelling factors. Two interleukins of great relevance to prostate cancer, IL6 and IL8, were further validated as EGR3 target genes: both promoters contain EGR consensus binding sites and are pulled down in intact cells by EGR3 chromatin immunoprecipitation. Silencing of EGR3 decreased IL6 and IL8 expression, whereas overexpression of EGR3 in nontransformed cells induced IL6 and IL8 expression.

Conclusions: Chronic inflammation plays a critical role in prostate cancer and elevated production of pro-inflammatory cytokines IL8 and IL6, in particular, contributes to disease progression and to the onset of castration resistance. It is shown for the first time that EGR3 is involved in the upregulation of both IL6 and IL8. Together with our previous observation that EGR3 is highly expressed in prostate tumours compared with normal tissue and strongly correlates with IL6 and IL8 expression in clinical samples, the present study suggests that EGR3 promotes excessive production of IL6 and IL8 observed during the progression of prostate cancer.

Inflammation is one of the hallmarks of cancer and plays an essential role in the development and progression of human tumours (Grivennikov et al, 2010; Hanahan and Weinberg, 2011; Balkwill and Mantovani, 2012). Thus, chronic inflammation increases cancer risk and many types of cancer arise at sites of inflammation (Balkwill et al, 2005; Ben-Baruch, 2006). It is thought to directly contribute to the initiation and the progression of prostate cancer, the second leading cause of cancer-related death in American men. Inflammation of the prostate is nearly ubiquitous in ageing men, triggered by a history of local viral or bacterial infection, diet, hormonal factors, systemic diseases such as obesity, and other environmental factors (De Marzo et al, 2007; De Nunzio et al, 2011). Epidemiological and clinical studies indicate that men with a history of prostatitis have an increased incidence of benign prostate hyperplasia or prostate cancer (reviewed in Sciarra et al, 2008), whereas the use of anti-inflammatory drugs such as aspirin is associated with reduced risks of prostate cancer (Pruthi and Wallen, 2005; Chan et al, 2006; Stock et al, 2008; Bardia et al, 2009; Harris, 2009). Finally, evidence of chronic inflammation in human specimen of benign prostate tissue was correlated with high-grade/

\footnotetext{
*Correspondence: Professor D Mercola; E-mail: dmercola@uci.edu

${ }^{3}$ Current address: Department of Statistics, University of Akron, Akron, OH 44325-1913 USA.

${ }^{4}$ Department of Family and Community Medicine, Northeast Ohio Medical University, Rootstown, OH 44272, USA.
}

Received 12 August 2014; revised 14 November 2014; accepted 20 November 2014; published online 29 January 2015 
aggressive prostate cancer, even in patients with low levels of prostate-specific antigen, underlying the critical role of inflammation in prostate cancer progression (Gurel et al, 2014).

There is now extensive evidence that chronic inflammation in the tumour environment inhibits the function of effector immune cells and suppresses the immune response against the tumour (Kim et al, 2007; Kanterman et al, 2012; Mittal et al, 2014). In his recent review of prostate cancer epidemiology, Brawley (2012) notes that up to $40 \%$ of surgically treated early-stage cancer cases (i.e., with intent to cure) do recur, suggesting that these tumours can indeed escape immune surveillance and become autonomous for growth and survival. Many of the immune cells that infiltrate the tumour promote its survival through the production of growth factors and proteases (Lin and Pollard, 2004). For example, B lymphocytes help maintain a state of chronic inflammation at the tumour site and promote carcinogenesis (de Visser et al, 2005). Infiltrating neutrophils activate angiogenesis (Nozawa et al, 2006) and increase metastasis by facilitating invasion through the basement membrane (Welch et al, 1989). Macrophages, which mediate initial tumour cytotoxicity and activate natural killer cells and $\mathrm{T}$ lymphocytes against tumour cells, are redirected toward protumour activities to produce angiogenic factors and growth factors, promote the degradation of the extracellular matrix and the invasion of tumour cells, and facilitate metastasis (reviewed in Lewis and Pollard, 2006; Mantovani et al, 2012).

The molecular mechanisms underlying the connection between sustained activation of inflammatory signalling pathways in prostate cancer and oncogenesis are not well understood. We report here that transcription factor EGR3 (Early Growth Response 3; also known as PILOT) regulates the expression of genes involved in inflammation, including genes encoding various secreted cytokines and protease inhibitors. As shown here, two of the cytokines that are induced by EGR3 in prostate cancer cells and were validated as direct target genes are interleukin 6 (IL6) and interleukin 8 (IL8), and these play major roles in the progression of prostate cancer (reviewed in Waugh and Wilson, 2008; Culig and Puhr, 2012). We have shown previously that in a set of 127 fresh frozen prostatectomy specimens with clinical outcome, EGR3 was highly expressed in prostate tumours compared with normal prostate samples and correlated with IL6 and IL8 expression (Pio et al, 2013). We propose therefore that high expression of EGR3 in human prostate tumours contributes to the elevated production of IL6 and IL8 by these tumours.

\section{MATERIALS AND METHODS}

Cell culture. Cell lines: P69 are immortalised nontransformed prostate epithelial cells and have been extensively characterised (Plymate et al, 1996); M12 are human prostate cancer cells developed as a tumourigenic derivative of P69 using serial passages of tumour xenografts (Bae et al, 1998). Cells were maintained in serum-free RMPI-1640 containing L-glutamine (10 $\mathrm{ng} \mathrm{ml})$, epidermal growth factor $(0.1 \mu \mathrm{M})$, dexamethazone, ITS $\left(5 \mu \mathrm{g} \mathrm{ml}^{-1}\right.$ each of insulin, transferring, and selenium), $0.05 \mathrm{mg} \mathrm{ml}^{-1}$ gentamicin, and $2.5 \mu \mathrm{g} \mathrm{ml}^{-1}$ amphotericin B.

Stable knockdown of EGR3 expression in M12 cells. M12 cells were plated the day before transfection at a density of 750000 cells per well in 6-well plates in antibiotic-free growth medium containing 2\% fetal bovine serum (FBS). Cells were transfected with $3 \mu \mathrm{g}$ shRNA scramble plasmid (shSCR) or shEGR3 plasmid (SA Biosciences, Qiagen, Valencia, CA, USA) with $6 \mu$ l Lipofectamine 2000 (Invitrogen, Carlsbad, CA, USA) according to the manufacturer's instructions. Puromycin $\left(1 \mu \mathrm{g} \mathrm{ml}^{-1}\right)$ was added 3 days later for selection of transfected cells. Single-cell clones were isolated using standard methods. Stably transfected cells
(shSCR-M12 and shEGR3-M12) were then maintained in growth medium supplemented with puromycin. Knockdown of EGR3 was stable over many cell culture passages. These cells have been previously described in Pio et al (2013).

Forced expression of EGR3 in P69 cells. The full-length EGR3 cDNA (clone 8327747) was purchased from Open Biosystems Inc. (GE Healthcare, Lafayette, CO, USA) and was excised from the pCR4-TOPO vector using the two flanking EcoRI sites. The EGR3 fragment was purified from agarose gel, treated with alkaline phosphatase, and inserted into the pcDNA3.1/V5-His-TOPO plasmid (Invitrogen Life Technologies, Grand Island, NY, USA). The orientation and sequence of the insert were verified by sequencing. Transfection with the empty plasmid or with the EGR3-containing plasmid was performed as described above. Selection was carried out in the presence of G418 $\left(0.2 \mathrm{mg} \mathrm{ml}^{-1}\right)$. Although cells were maintained in the presence of G418, expression of EGR3 was not stable over time and only early passage cells were used.

Western blot analysis. Cell lysis and western blots were carried out as described previously (Pio et al, 2013). EGR3 antibodies (sc-191) and $\beta$-actin antibodies were purchased from Santa Cruz Technology (Santa Cruz, CA, USA). In some experiments, EGR3 antibody from Cell Signaling Technology (Danvers, MA, USA) was used. Some of the western blots were quantified using ImageJ, a public domain Java image processing program (http://imagej.nih. gov/ij/) (Schneider et al, 2012).

RNA purification and cDNA synthesis. Using the RNeasy Plus kit (Qiagen, Valencia, CA, USA), RNA was extracted from M12 or P69 cells. The RNA integrity was assessed by electrophoresis on formaldehyde agarose gels and the RNA was quantified using the Beckman Du800 spectrophotometer (Indianapolis, IN, USA). Using the Quanta qScript cDNA Super-mix $\left(5 \mathrm{~min}\right.$ at $25^{\circ} \mathrm{C} ; 30 \mathrm{~min}$ at $42^{\circ} \mathrm{C}$; $5 \mathrm{~min}$ at $\left.85^{\circ} \mathrm{C}\right), 1 \mu \mathrm{g}$ RNA was converted to cDNA.

Affymetrix gene expression analysis. Hybridisation to Affymetrix (Santa Clara, CA, USA) U133Plus2.0 arrays was performed using biological duplicates for each sample (shSCR-M12, shEGR3-M12 clone 2, and shEGR3-M12 clone 3, e.g., 6 arrays). Duplicates were collected on different days to account for day-to-day variation. In addition to electrophoresis, the excellent quality of the RNA was confirmed using an Agilent (Santa Clara, CA, USA) Bioanalyzer 2100 before hybridisation on Affymetrix chips. The University of California Genomics High Throughput Facility carried out the in vitro transcription according to their standard protocol (http:// ghtf.biochem.uci.edu) and hybridised the biotinylated cRNA to Affymetrix human genome U133Plus2.0 gene expression arrays. These arrays contain 54000 probe sets that cover the whole human genome. The raw Affymetrix expression values were normalised and analysed for differential expression using the Limma package of the R program (http://www.r-project.org/). The $t$-tests were performed to establish $P$-values for differential expression. Fold changes of $\geqslant 2$ and $\leqslant-2$ were considered valid and $P$-values of $\leqslant 0.01$ were considered statistically significant.

Gene expression data have been deposited in the Gene Expression Omnibus (GEO) database as GSE52108 (Series Accession: GSE52108 ID: 200052108).

Quantitative RT-PCR. Real-time qPCR was performed on the ABI Prism 7900HT (Applied Biosystems, Life Technologies, Grand Island, NY, USA) using standard parameters ( $4 \mathrm{~min}$ at $95^{\circ} \mathrm{C}$ followed by 40 cycles of $15 \mathrm{~s}$ at $95^{\circ} \mathrm{C}$ and $60 \mathrm{~s}$ at $60^{\circ} \mathrm{C}$ ). Each sample was run in four replicates with dissociation curve analysis. Differences in mRNA levels were analysed using the 2- $\triangle \Delta \mathrm{CT}$ method. Glyceraldehyde-3-phosphate dehydrogenase (GAPDH) was used to normalise samples. 
Promoter analysis. Gene promoter sequences were downloaded from the University of California Santa Cruz Genome Browser (http://genome.ucsc.edu/cgi-bin/hgGateway) based on the February 2009 human reference sequence. Genomic sequences from 1500 base-pair upstream to 1000 base-pair downstream of the transcription start site were analysed. The Genomatix MatInspector software and the University of Pennsylvania Transcription Element Search System (TESS, http://www.cbil.upenn.edu/cgi-bin/tess) were used to search sequences corresponding to potential EGR-binding sites as identified by the TRANSFAC database.

\section{RESULTS}

We were first to report that EGR3 is overexpressed in clinical human prostate cancer compared with normal tissue (Pio et al, 2013). However, its role in prostate cancer is completely unknown. To understand the function of EGR3, we looked for human prostate cancer cells lines that contain high levels of EGR3. Publicly available data set GDS3155 provides gene expression data for 15 prostate cancer cell lines (Wang et al, 2007) and was downloaded to extract EGR3-related data. As shown in Figure 1A and B, EGR3 expression is mostly similar across cell lines with the exception of HPV7 and HPV10 cell lines, and is comparable to the expression of EGR2 and EGR4. Family member EGR1, in contrast, displays high variability in expression across cell lines (Figure 1B).

Western blot analysis of EGR3 protein levels in various cell lines identified an isogenic pair of prostate epithelial cells with distinctive EGR3 expression. Human prostate cells P69 are nontransformed immortalised prostate epithelial cells, whereas M12 cells were developed as a tumourigenic derivative of P69 cells after serial passages as tumour xenografts (Bae et al, 1998). Isogenic cells are useful to perform gain-of-function and loss-offunction studies because comparisons between the cells are not confounded by genetic differences. Thus, the prostate cancer cell line M12, not represented in the GDS3155 data set, displays high levels of EGR3 protein compared with cell line P69 (Figure 1C). This was confirmed at the mRNA levels measured by real-time qPCR (Figure 1D), indicating that M12 cells contain $\sim 5$ times more EGR3 mRNA than P69 cells. In addition, it was observed that EGR3 is almost entirely nuclear in M12 cells, as would be expected of an active transcription factor (Figure 1E). Interestingly, none of the cell lines analysed in GDS3155 really corresponds to a primary tumour. Cells lines used in this gene expression study were generated through immortalisation of noncancer cells using HPV or SV40-T antigen, through chemically induced transformation
A

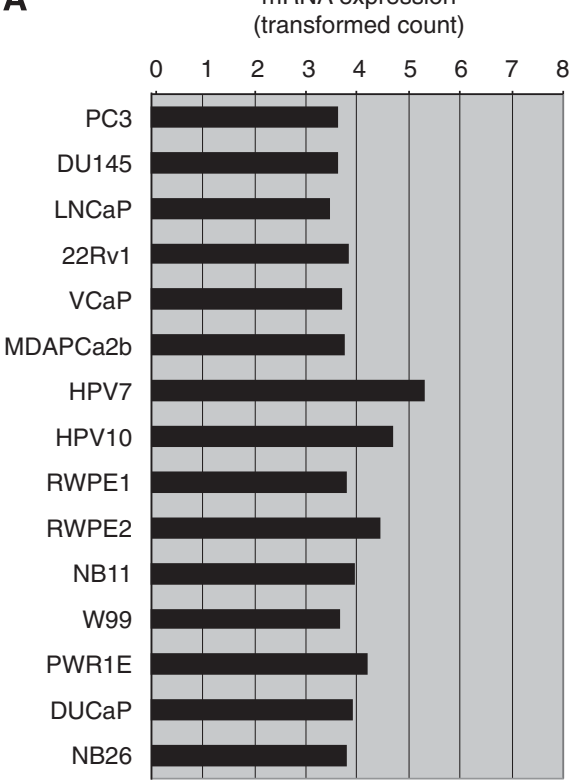

C

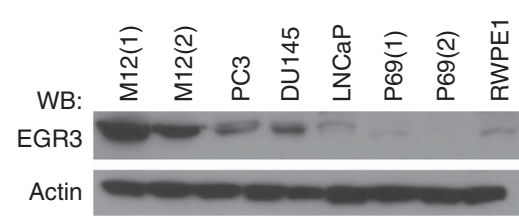

B

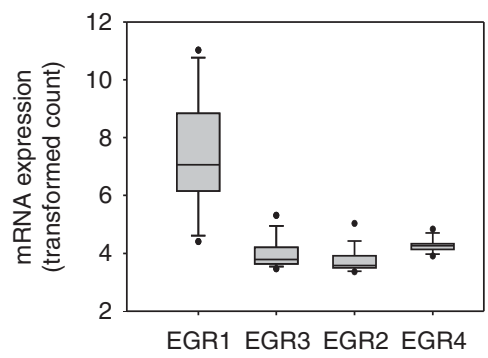

D

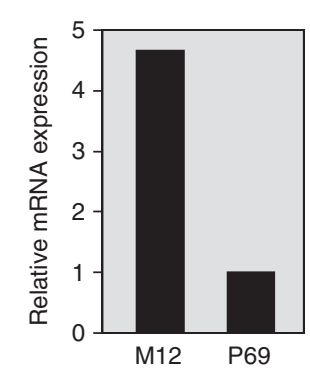

E Fraction: $\mathrm{C} \mathrm{N}$

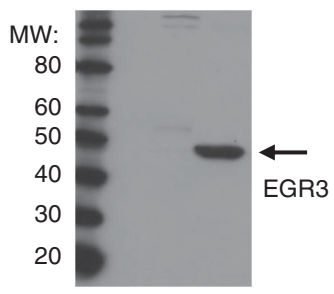

Figure 1. EGR3 expression in human prostate cell lines. (A) The EGR3 gene expression values were retrieved from GEO data set GDS3155 that contains expression data for 15 human prostate epithelial cell lines (note that the stroma cell line WPMY present in the data set was excluded from this figure). (B) Box plot of the mRNA expression of EGR family members in this data set, reflecting high variability (EGR1) and low variability of expression (EGR2-4). (C) EGR3 protein expression in a few prostate cell lines. Untreated cells were lysed and protein expression was analysed by western blot using anti-EGR3 antibodies. The EGR3 protein migrates at $\sim 50 \mathrm{kDa}$. Membranes were reprobed with anti-actin antibodies as loading control. (D) Comparison of EGR3 mRNA expression in M12 and P69 cells. Total RNA was extracted from untreated cells and EGR3 expression was measured by quantitative RT-PCR. Results are expressed relative to expression in P69 cells. (E) Subcellular localisation of EGR3 in M12 prostate cancer cells. Untreated prostate cancer cells M12 were lysed and fractionated using a commercial kit, followed by western blot analysis of protein levels using anti-EGR3 antibodies. 
in vitro, or through isolation from human metastases. In contrast, the M12 prostate cancer cells having been isolated from a xenograft tumour grown in mice may better correspond to a primary tumour, at least in terms of EGR3 expression. Early Growth Response 3 belongs to the EGR family of transcription factors that also includes EGR1, and is of particular interest because it also plays a role in prostate cancer. To determine whether EGR3 is induced similarly or differently than EGR1, M12 and P69 cells were treated with TPA (12-O-tetradecanoylphorbol-13-acetate) or FBS for increasing times. These treatments are known to strongly induce the expression of EGR1. As shown in Figure 2A and B, EGR3 expression was not altered by either TPA or FBS, although both treatments increased EGR1 expression in both cell lines. The EGR3 protein was barely detectable in P69 cells, whereas it was highly expressed in M12 independently of treatment. Thus, EGR3 is not induced by TPA or by FBS in contrast to EGR1, indicating that the two transcription factors are differently activated in prostate cells and have different functions.

In another set of experiments, conditioned medium was collected from M12 cultures and applied to P69 cells for up to $24 \mathrm{~h}$. As shown in Figure 2C, EGR3 was induced following treatment of P69 cells with M12 conditioned medium. The effect was maximum after $24 \mathrm{~h}$ as indicated by quantification of the blot. Lysates from M12 cells were loaded on the gel as a positive control (last lane). This experiment indicates that P69 cells contain a functional EGR3 that can be induced by soluble cytokines. It also suggests that M12 cells secrete EGR3-inducing cytokines and that high expression of EGR3 in these cells may be the result of autocrine activation.

EGR3 silencing and gene expression analysis. The M12 human prostate cancer cells were therefore chosen to study the function of EGR3 using shRNA silencing. Cells were transfected with a scrambled control shRNA plasmid (shSCR) or shEGR3 plasmid and selected with puromycin as described previously (Pio et al, 2013). Several clones were isolated, two of which (termed shEGR3cl 2 and shEGR3-cl3) displayed an average $40 \%$ to $50 \%$ inhibition of EGR3 protein expression compared with the shSCR-M12 control cells and were used for further study (Figure 3A and B). Genes that are regulated by transcription factor EGR3 in these cells were identified by genome-wide expression analysis comparing control shSCR-M12 with shEGR3-cl2 and shEGR3-cl3. Genes with differences of expression $\geqslant 2$-fold (up or down) and with $P \leqslant 0.01$ were considered differentially regulated. Out of the 54000 probes in the Affymetrix U133Plus2.0 arrays, the overlap between the two shEGR3 clones consists of 332 differentially expressed genes most likely to be regulated by EGR3. Table $1 \mathrm{~A}$ displays the top 20 genes that were induced by EGR3 in M12 cells (i.e., genes for which expression was lower in shEGR3-M12-silenced cells compared with shSCR-M12 control cells), and Table 1B displays the top 20 genes that were repressed by EGR3 in M12 cells (i.e., genes for which expression was higher in shEGR3-M12-silenced cells compared with shSCR-M12 control cells), respectively. The full list of differentially expressed genes can be found in Supplementary Tables S1 and S2.

An extensive simulation study was performed to determine whether the 332 gene overlap between the two clones was likely to occur by chance. In 10000 simulations in which the lists were assigned genes at random, an overlap of 332 genes or more was never found $(P<0.0001)$, indicating that the intersection of Clone 2 and Clone 3 is statistically significant. The list of EGR3regulated genes is indeed very robust (Huang da et al, 2009a): (1) duplicate arrays were used for each sample; (2) biological reproducibility was ensured by the use of two independent clones, limiting the so-called 'clonal effects'; (3) the list contains a reasonable number of genes; (4) strong statistical thresholds were used for selection (cutoff of two-fold changes with $P \leqslant 0.01$ ); and
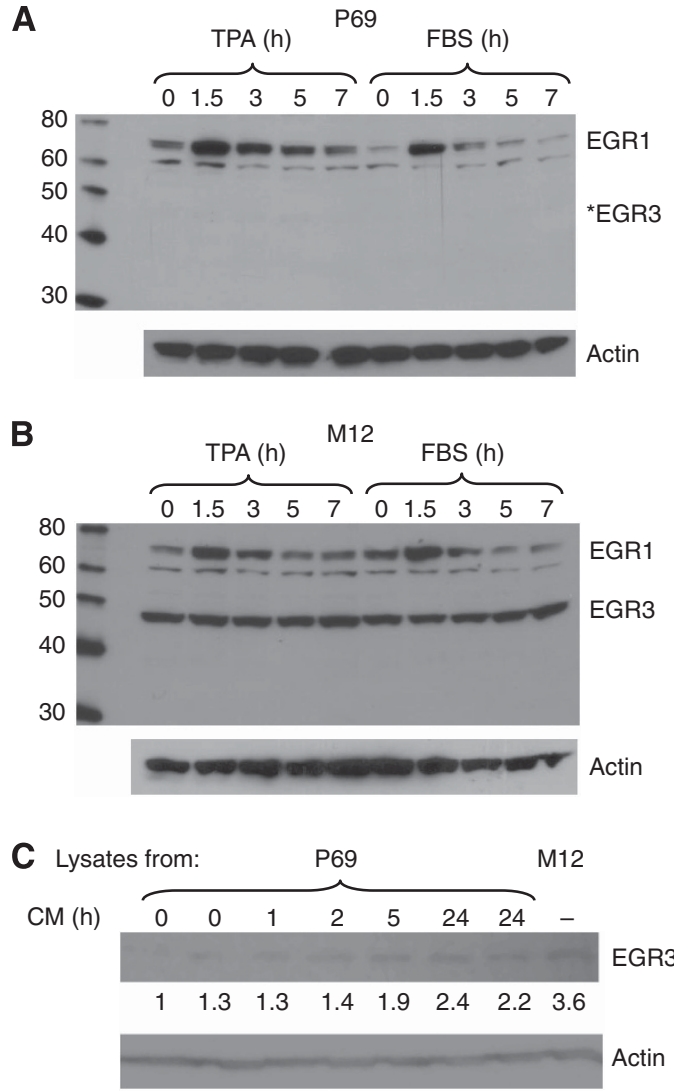

Figure 2. Inducibility of EGR3 in M12 and P69 prostate epithelial cells. Cells were treated with TPA $\left(10 \mathrm{ng} \mathrm{ml}^{-1}\right)$ or FBS (10\%) for the indicated times. The protein expression levels of EGR3 and EGR1 were measured by western blot using successively EGR3 and EGR1 antibodies (without stripping). The EGR1 was used as a positive control for the effect of TPA and FBS on the expression of early response genes. Membranes were then stripped and reprobed with anti-actin antibodies as loading control. (A) P69 cells. The symbol (*) denotes the position of EGR3 if it was present. (B) M12 cells. (C) Conditioned medium (CM) from M12 cells was applied to P69 cells for the indicated times. The P69 cells were lysed and EGR3 protein levels were measured by western blot. Cell lysates from untreated M12 cells were used as positive control (far right lane). Note that the signal intensity for EGR3 is different than in other western blots because a different commercial antibody was used.
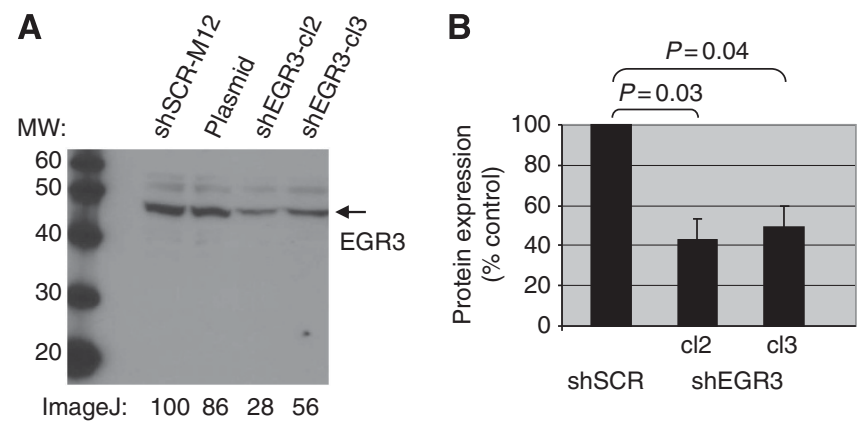

Figure 3. The shRNA-mediated EGR3 silencing in M12 cells. (A) The M12 cells were stably transfected with the empty vector, a scramble control shRNA (shSCR-M12), or EGR3-shRNA (shEGR3-clone 2 and shEGR3-clone 3). Protein expression of EGR3 was analysed by western blotting at various cell passages. A representative western blot is shown. (B) Western blots from three separate experiments (at three different cell culture passages) were quantified using ImageJ processing software. The graph represents the average of EGR3 expression compared with $\mathrm{shSCR} \pm \mathrm{S}$.E. 
Table 1A. Top 20 genes that are induced by EGR3 in M12 cells (i.e., genes for which expression was lower in shEGR3-M12silenced cells compared with shSCR-M12 control cells)

\begin{tabular}{|c|c|c|c|c|c|}
\hline & Gene name & Gene symbol & Probe ID & Fold change & $P$-value \\
\hline 1 & Pentraxin-related gene, rapidly induced by IL-1 $\beta$ & PTX3 & 206157_at & -105.94 & $6 \mathrm{E}-09$ \\
\hline 2 & Oxidised low-density lipoprotein (lectin-like) receptor 1 & OLR1 & 210004_at & -100.22 & $2 \mathrm{E}-09$ \\
\hline 3 & Keratin 34 & KRT34 & 206969_at & -86.04 & $3 E-09$ \\
\hline 4 & Microfibrillar associated protein 5 & MFAP5 & $\begin{array}{c}\text { 209758_s_at } \\
\text { 213764_s_at } \\
213765 \text { at }\end{array}$ & $\begin{array}{l}-85.61 \\
-27.52 \\
-14.57\end{array}$ & $\begin{array}{l}4 E-08 \\
6 E-09 \\
9 E-08\end{array}$ \\
\hline 5 & Monoamine oxidase $\mathrm{A}$ & MAOA & $\begin{array}{c}\text { 204388_s_at } \\
\text { 204389_at } \\
212741 \text { at }\end{array}$ & $\begin{array}{l}-67.5 \\
-37.76 \\
-37.24\end{array}$ & $\begin{array}{l}2 E-09 \\
1 E-07 \\
2 E-07\end{array}$ \\
\hline 6 & Chemokine (C-X-C motif) ligand 1 (melanoma growth stimulating activity, $\alpha$ ) & CXCL1 & 204470_at & -57.03 & $7 E-08$ \\
\hline 7 & Regulating synaptic membrane exocytosis 2 & RIMS2 & $\begin{array}{l}\text { 229823_at } \\
\text { 206137_at }\end{array}$ & $\begin{array}{l}-49.26 \\
-15.91\end{array}$ & $\begin{array}{l}1 E-09 \\
5 E-07\end{array}$ \\
\hline 8 & Gap junction protein, $\alpha 1,43 \mathrm{kDa}$ & GJA1 & 201667_at & -41.7 & $3 E-08$ \\
\hline 9 & Tissue factor pathway inhibitor 2 & TFPI2 & $\begin{array}{c}\text { 209278_s_at } \\
\text { 209277_at }\end{array}$ & $\begin{array}{c}-36.22 \\
-8.44\end{array}$ & $\begin{array}{l}6 E-09 \\
8 E-06\end{array}$ \\
\hline 10 & Membrane metallo-endopeptidase & MME & $\begin{array}{l}\text { 203434_s_at } \\
\text { 203435_s_at }\end{array}$ & $\begin{array}{c}-28.88 \\
-6.27\end{array}$ & $\begin{array}{l}3 E-06 \\
2 E-07\end{array}$ \\
\hline 11 & Complement component 4 binding protein, $\beta$ & C4BPB & 208209_s_at & -23.48 & $9 E-08$ \\
\hline 12 & Doublecortin and CaM kinase-like 1 & DCAMKL1 & 205399_at & -22.96 & $6 E-09$ \\
\hline 13 & Lymphotoxin $\beta$ (TNF superfamily, member 3) & LTB & 207339_s_at & -19.29 & $1 \mathrm{E}-08$ \\
\hline 14 & Chemokine ( $\mathrm{C}-\mathrm{C}$ motif) ligand 20 & CCL20 & 205476_at & -18.71 & $6 \mathrm{E}-08$ \\
\hline 15 & A kinase (PRKA) anchor protein 12 & AKAP12 & $\begin{array}{c}\text { 227529_s_at } \\
\text { 227530_at } \\
\text { 210517_s_at } \\
\text { 231067_sat }\end{array}$ & $\begin{array}{c}-18.38 \\
-14.76 \\
-11.06 \\
-3.4\end{array}$ & $\begin{array}{l}5 E-08 \\
3 E-08 \\
7 E-07 \\
0.0001\end{array}$ \\
\hline 16 & Chemokine (C-X-C motif) ligand 6 (granulocyte chemotactic protein 2) & CXCL6 & 206336_at & -18.11 & $2 E-06$ \\
\hline 17 & Doublecortin-like kinase 1 & DCLK1 & 229800_at & -17.89 & $2 E-08$ \\
\hline 18 & DENN/MADD domain containing $4 B$ & DENND4B & 202860_at & -17.54 & $2 \mathrm{E}-07$ \\
\hline 19 & Interleukin 8 & IL8 & 211506_s_at & -17.34 & $3 \mathrm{E}-07$ \\
\hline 20 & Protocadherin 7 & $\mathrm{PCDH7}$ & $\begin{array}{l}\text { 205534_at } \\
228640 \text { at }\end{array}$ & $\begin{array}{l}-17.22 \\
-12.83\end{array}$ & $\begin{array}{l}2 E-07 \\
4 E-07\end{array}$ \\
\hline
\end{tabular}

(5) the list of genes is strongly enriched in functional terms (described below).

Biological meaning was extracted from the list of EGR3regulated genes using publicly available bioinformatics resource DAVID http://david.abcc.ncifcrf.gov/ (Huang da et al, 2009a, b). The most striking feature of this analysis is the high representation of genes related to inflammation and the immune system as well as genes with a role in the regulation of the extracellular environment (Supplementary Table S3).

Thus, two of the three disease-related terms found in the functional annotation chart are 'Immune disease' and 'Infection', with a count of 48 genes ( $P$-value $2.24 \mathrm{E}-05)$ and 22 genes $(P$-value $=0.0011)$, respectively. Approximately $35 \%$ of the terms in the functional categories chart are related to immune responses and inflammation, whereas another $35 \%$ are related to the regulation of the microenvironment. This was also evident from the functional annotation clustering, shown in Supplementary Table S4. The terms 'response to wounding' $(P$-value $1.96 \mathrm{E}-14)$, 'inflammatory response' ( $P$-value $2.97 \mathrm{E}-10)$, and 'defense response' $(P$-value $=6.58 \mathrm{E}-10)$ had an enrichment score of 10.8 .

Interestingly, 61 of the EGR3-regulated genes (i.e., 18.7\%) encode secreted proteins (Supplementary Table S5). This indicates that EGR3 activation in prostate cancer cells may alter the tissue microenvironment through the secretion of inflammatory cytokines and growth factors, extracellular matrix proteins, insulin-like growth factor (IGF)-binding proteins, and protease inhibitors (notably of the Serpin family of peptidase inhibitors SERPINE1,
SERPINB1-B2-B7-B9), whereas the expression of extracellular proteases was repressed by EGR3 (e.g., PCSK5, PRSS3). In addition, a set of 18 genes regulated by EGR3 (cluster 14, Supplementary Table S2) are involved in the positive regulation of transport and secretion, consistent with an increased usage of the cellular machinery involved in the production of secreted proteins.

A total of 20 genes encoding cytokines or cytokine receptors were regulated by EGR3 in M12 cells (i.e., were decreased in shEGR3-M12 cells compared with control cells): CXCL1, CXCL2, CXCL3, CXCL5, CXCL6, CCL5, CCL20, IL6, IL8, IL15, IL23A, IL1A, IL6ST(gp130), IL7R, IL1R2, BMP2, CSF2, LTB, TNFSF18, and $I N H B A$. Especially interesting may be the induction of ligand/ receptor pairs, as it suggests the establishment of potential autocrine loops. For example, IL6 and IL6ST are respectively 6-fold and 3-fold higher in control M12 cells than in shEGR3silenced cells, whereas IL1A and its receptor IL1R2 are respectively 12-fold and 5.6-fold higher.

The pathway software GeneGo (Metacore, Thompson Reuters, New York, NY, USA) was also used to group our list of genes into functional categories according to transcription factor networks, canonical pathways, gene ontology terms, and so on (summarised in Table 2). Again, inflammation and immune processes were the most represented. Many of the genes present on our list map to canonical inflammatory pathways such as interleukin-17 (IL17)-, IL6-, and IL1-mediated signalling pathways ( $P$-values for the enrichment of genes in the IL17 and IL1/IL6 pathways are $3.61 \times 10^{-9}$ and $3.56 \times 10^{-5}$, respectively). 
Table 1B. Top 20 genes that are repressed by EGR3 in M12 cells (i.e., genes for which expression was higher in shEGR3-M12silenced cells compared with shSCR-M12 control cells)

\begin{tabular}{|c|c|c|c|c|c|}
\hline & Gene name & Gene symbol & probe ID & Fold change & $P$-value \\
\hline 1 & Colorectal neoplasia differentially expressed (non-protein coding) & CRNDE & 1552797_s_at & 32.28 & $2 \mathrm{E}-08$ \\
\hline 2 & H19, imprinted maternally expressed transcript (non-protein coding) & H19 & $\begin{array}{l}\text { 1562019_at } \\
\text { 200884_at }\end{array}$ & $\begin{array}{l}24.59 \\
22.93\end{array}$ & $\begin{array}{l}2 \mathrm{E}-08 \\
8 \mathrm{E}-09\end{array}$ \\
\hline 3 & S100 calcium binding protein A2 & S100A2 & 201331_s_at & 11.6 & $8 \mathrm{E}-08$ \\
\hline 4 & Cytochrome P450, family 4, subfamily X, polypeptide 1 & CYP4X1 & 201641_at & 10.48 & $6 E-08$ \\
\hline 5 & Protease, serine, 3 & PRSS3 & $\begin{array}{l}\text { 201820_at } \\
\text { 202437_s_at }\end{array}$ & $\begin{array}{l}8.784 \\
8.062\end{array}$ & $\begin{array}{l}3 \mathrm{E}-07 \\
8 \mathrm{E}-08\end{array}$ \\
\hline 6 & Phosphatidic acid phosphatase type 2 domain containing $1 \mathrm{~A}$ & PPAPDC1A & 202504_at & 8.618 & $2 E-06$ \\
\hline 7 & Solute carrier family 2 (facilitated glucose transporter), member 10 & SLC2A10 & 203381_s_at & 7.59 & $3 E-05$ \\
\hline 8 & Desmoglein 3 (pemphigus vulgaris antigen) & DSG3 & 203382_s_at & 6.705 & $4 E-05$ \\
\hline 9 & Bone morphogenetic protein 2 & BMP2 & $\begin{array}{c}\text { 203543_s_at } \\
\text { 203729_at }\end{array}$ & $\begin{array}{l}6.648 \\
5.956\end{array}$ & $\begin{array}{l}2 E-06 \\
9 E-06\end{array}$ \\
\hline 10 & Sialic acid acetylesterase & SIAE & 204225_at & 5.906 & $3 E-06$ \\
\hline 11 & $\begin{array}{l}\text { Potassium large conductance calcium-activated channel, subfamily } M, \alpha \\
\text { member } 1\end{array}$ & KCNMA1 & 204268_at & 5.868 & $5 E-07$ \\
\hline 12 & Sulfotransferase family $1 E$, oestrogen-preferring, member 1 & SULT1E1 & 204455_at & 5.236 & $4 E-06$ \\
\hline 13 & Spleen tyrosine kinase & SYK & $\begin{array}{l}\text { 204537_s_at } \\
\text { 204540_at }\end{array}$ & $\begin{array}{l}5.147 \\
4.367\end{array}$ & $\begin{array}{l}1 \mathrm{E}-05 \\
1 \mathrm{E}-06\end{array}$ \\
\hline 14 & Growth factor receptor-bound protein 10 & GRB10 & 204783_at & 4.709 & $1 \mathrm{E}-05$ \\
\hline 15 & S100 calcium binding protein A14 & S100A14 & 204955_at & 4.474 & $3 E-06$ \\
\hline 16 & Chemokine (C-X-C motif) receptor 7 & CXCR7 & 205128_x_at & 4.365 & $8 \mathrm{E}-05$ \\
\hline 17 & Shisa homolog 2 (Xenopus laevis) & SHISA2 & 205157_s_at & 4.321 & $5 E-05$ \\
\hline 18 & $\begin{array}{l}\text { UDP glucuronosyltransferase } 1 \text { family, polypeptide A3; polypeptide A5; } \\
\text { polypeptide A4; polypeptide A7; polypeptide A6; polypeptide A10; } \\
\text { polypeptide A9; polypeptide A8; polypeptide A1 }\end{array}$ & UGT1A & $\begin{array}{l}\text { 205289_at } \\
\text { 205290_s_at } \\
\text { 205319_at } \\
\text { 205559_s_at }\end{array}$ & $\begin{array}{l}3.242 \\
3.074 \\
2.656 \\
\end{array}$ & $\begin{array}{l}6 E-06 \\
1 E-05 \\
3 E-06 \\
2 E-05 \\
\end{array}$ \\
\hline 19 & DEAD (Asp-Glu-Ala-Asp) box polypeptide 60 & DDX60 & 205730_s_at & 4.269 & $6 E-07$ \\
\hline 20 & Prostate stem cell antigen & PSCA & 205885_s_at & 4.061 & $3 E-06$ \\
\hline
\end{tabular}

A fairly large number of EGR3-regulated genes in M12 cells (53 out of 247) also map to the NF- $\kappa \mathrm{B}$ pathway (e.g., RELB, $N F K B I Z$, and NFKBIE) and our gene list was very significantly enriched with NF- $\kappa \mathrm{B}$-interacting genes $\left(P\right.$-value $=8.96 \times 10^{-24}$ based on a false discovery rate of 0.05 ). The map of NF- $\kappa \mathrm{B}$ interacting genes that are regulated by EGR3 is shown in Supplementary Figure S1. NF- $\kappa \mathrm{B}$ is a well-known regulator of inflammation (reviewed in Karin, 2009). Early Growth Response 3 and NF- $\kappa \mathrm{B}$ may act as cofactors in the regulation of various inflammatory genes. For example, EGR3 interacts with $\mathrm{NF}-\kappa \mathrm{B}$ subunit p65 in intact cells, and the complex strongly activates the promoters of IL2, tumour necrosis factor- $\alpha$ (TNF $\alpha)$, and intercellular cell adhesion molecule-1 (ICAM1) (Wieland et al, 2005). This cooperation may be important for the expression of inflammatory genes by prostate tumour cells and it will be interesting, in the future, to see how blocking the NF- $\kappa \mathrm{B}$ pathway would affect EGR3-induced gene expression.

EGR3 triggers the expression of IL6 and IL8. The list of EGR3induced genes obtained in the present study was compared with a previously published list of genes for which expression highly correlated with that of EGR3 in 91 clinical human prostate cancer specimens (Pio et al, 2013). We hypothesised that there could be a biological relevance to the fact that EGR3 is upregulated in human prostate cancer samples compared with normal tissue samples, and is also highly expressed in M12 cells that were originally derived from a mouse tumour. Many genes that correlate with EGR3 in human tumour samples are not directly regulated by EGR3 and the overlap is not expected to be large; however, a Venn diagram may highlight genes that are regulated by EGR3 both in vitro (cancer cells) and in vivo (human clinical samples) and play important roles in prostate cancer. Nine genes were found at the intersection of the two lists (Figure 4A), among which are $H B E G F$ and cytokine genes CXCL2, IL8, and IL6.

In our previous study of human prostate cancer samples, the expression of IL6 and IL8 showed a very strong correlation with the expression of EGR3 (Pio et al, 2013). Indeed, IL6 and EGR3 correlation factor was 0.603 and 0.774 in the clinical data sets (slope 1.052; $n=263$ and $P \leqslant 0.001$ ), whereas IL8 and EGR3 correlation factor was 0.633 and 0.536 (slope $0.738 ; n=263$ and $P \leqslant 0.001$ ). In this study a multiple linear regression model had been used to integrate gene expression levels and cell type composition of the samples (four cell types), as initially described in Wang et al (2010), yielding cell type-specific expression coefficients. This allowed us to calculate gene expression correlations within the epithelial cell type with reasonable accuracy. However, we cannot rule out that increased expression of some of the genes that correlate with EGR3 in human samples such as IL6 and IL8 may be contributed, at least in part, by infiltrating immune cells.

Both IL6 and IL8 play a major role in prostate cancer progression but the molecular mechanism leading to their high expression in human prostate cancer is still incompletely understood, and therefore we focused on their validation as potential EGR3 target genes. A small-scale real-time qPCR analysis shown in our previous study indicated that the expression of IL6 and IL8 
Table 2. GeneGo enrichment of EGR3-regulated genes

\begin{tabular}{|c|c|c|c|}
\hline GeneGo pathway maps & $P$-value & Gene ontology processes & $P$-value \\
\hline Cytokine production by Th17 cells in CF & $1.1 \mathrm{E}-09$ & Cellular response to chemical stimulus & $1.5 \mathrm{E}-26$ \\
\hline Immune response_IL-17 signalling pathways & $3.6 \mathrm{E}-09$ & Immune system process & $2.2 \mathrm{E}-24$ \\
\hline Cytokine production by Th17 cells in CF (mouse) & $1.7 \mathrm{E}-07$ & Response to chemical stimulus & $4.7 \mathrm{E}-21$ \\
\hline Immune response_Histamine $\mathrm{H} 1$ receptor & $2.3 \mathrm{E}-06$ & Response to organic substance & $6.8 \mathrm{E}-21$ \\
\hline Androstenedione and testosterone biosynthesis & $4.7 \mathrm{E}-06$ & Defense response & $1.2 E-16$ \\
\hline Androstenedione and testosterone biosynthesis & $5.6 \mathrm{E}-06$ & Cellular response to organic substance & $1.2 \mathrm{E}-15$ \\
\hline Immune response_IL-1 signalling pathway & $1.9 E-05$ & Immune response & $1.5 E-15$ \\
\hline Immune response_Innate immune response to RNA viral infection & $2.5 \mathrm{E}-05$ & Negative regulation of biological process & $7.0 \mathrm{E}-15$ \\
\hline Immune response_Signalling pathway mediated by IL- 6 and IL-1 & $3.6 \mathrm{E}-05$ & Response to wounding & $1.3 \mathrm{E}-14$ \\
\hline Cell adhesion_Chemokines and adhesion & $4.1 \mathrm{E}-05$ & Response to cytokine stimulus & 1.7E -14 \\
\hline GeneGo process networks & $P$-value & GeneGo metabolic networks & $P$-value \\
\hline Cell adhesion_Platelet-endothelium-leukocyte interactions & $6.2 \mathrm{E}-11$ & O-hexanoyl-(L)-carnitine pathway & $1.8 \mathrm{E}-03$ \\
\hline Development_Regulation of angiogenesis & $9.8 \mathrm{E}-10$ & Acyl-L-carnitine pathway & $1.8 \mathrm{E}-03$ \\
\hline Inflammation_Interferon signalling & $5.5 \mathrm{E}-08$ & Myristoyl-L-carnitine pathway & $2.3 E-03$ \\
\hline Immune response_Innate immune response to RNA viral infection & $1.0 \mathrm{E}-06$ & Stearoylcarnitine pathway & $2.7 E-03$ \\
\hline Inflammation_IFN- $\gamma$ signalling & $2.7 \mathrm{E}-06$ & 6'-sialyllactose pathways and transport & $2.9 E-03$ \\
\hline Immune response_Th17-derived cytokines & $5.5 \mathrm{E}-06$ & Lyso-Phosphatidylserine pathway & $6.7 \mathrm{E}-03$ \\
\hline Inflammation_MIF signalling & $6.4 \mathrm{E}-06$ & Maltohexaose pathways and transport & $9.8 \mathrm{E}-03$ \\
\hline Inflammation_Protein C signalling & $1.4 \mathrm{E}-05$ & Maltopentaose pathways and transport & $1.3 \mathrm{E}-02$ \\
\hline Blood coagulation & $2.4 \mathrm{E}-05$ & GalNAcbeta1-3Gal pathway & $1.3 \mathrm{E}-02$ \\
\hline Inflammation_Innate inflammatory response & $2.5 \mathrm{E}-05$ & Steroid metabolism_Estrone and Estradiol met. & $1.3 \mathrm{E}-02$ \\
\hline
\end{tabular}

were indeed reduced in shEGR3-M12-silenced cells. Figure 4B extends this analysis to confirm that seven other genes chosen at random from the list of EGR3-regulated genes were decreased upon EGR3 silencing, in addition to IL6 and IL8.

The promoters of both IL6 and IL8 indeed contain putative EGR-binding sites, as highlighted in Supplementary Figure S2, making them good candidates to be EGR3 targets. Several lines of evidence validate this hypothesis. One was that EGR3 silencing decreased the activity of a reporter gene, as shown previously (Pio et al, 2013). More importantly, we now show using chromatin immunoprecipitation that EGR3 directly binds to the promoter of IL6 and IL8 genes in intact cells (Figure 4C), consistent with the notion that it directly controls their expression.

The effect of EGR3 overexpression was then explored in the P69 cells that are isogenic to M12 but contain low basal levels of EGR3 compared with M12. A tagged EGR3 expression plasmid or the empty vector were transfected into P69 cells, resulting in a cell line that displayed strong overexpression of EGR3 (Figure 5A). As had been observed in M12 cells, the overexpressed EGR3 protein in P69 cells was mostly nuclear (Figure 5B). The expression of IL6 and IL8, in addition to several genes chosen at random from the list of EGR3-correlated genes, were compared in control P69 cells and in EGR3-transfected P69 cells. Among the genes tested, only IL6 and IL8 were directly induced by EGR3 in the transfected P69 cells (Figure 5C) and this provides further demonstration that both IL6 and IL8 are under the control of EGR3 in prostate epithelial cells.

As a whole, data presented here indicate that EGR3 regulates the expression of inflammation genes and increases the expression of cytokines IL6 and IL8. Because EGR3 is highly expressed in human prostate tumour tissue compared with normal tissue, we propose that it contributes to the overexpression and secretion of IL6 and IL 8 by prostate tumours.

\section{DISCUSSION}

Early Growth Response 3 belongs to the EGR family of transcription factors with critical functions in the brain (Perez-Cadahia et al, 2011) and immune cells (Gomez-Martin et al, 2010). It may play a role in cancer as well, although evidence remains scant. It has been shown that oestrogen induces EGR3 in breast cancer cells (Inoue et al, 2004), and that prolactin together with oestrogen synergistically increases EGR3 expression (Rasmussen et al, 2010). In human breast tumour tissue, EGR3 expression was positively associated with the expression of oestrogen receptor $\alpha$, with lymph node invasion and metastasis, and was associated with adverse outcome (Suzuki et al, 2007). In addition, forced expression of EGR3 in breast cancer cells increased cell invasion in vitro and in vivo (Suzuki et al, 2007). There is also recent evidence that EGR3 expression is lower in gastric cancer tissue compared with normal tissue, and that lower expression in gastric cancer is associated with adverse outcome (Liao et al, 2013). Finally, we previously observed that EGR3 expression in prostate cancer is significantly higher in clinical human tumour tissue compared with normal prostate tissue at both mRNA and protein levels (Pio et al, 2013). The EGR3 expression was generally higher in epithelial cells than in stroma cells in our data set, and this was confirmed in another publicly available data set that contains stroma/epithelial data as shown in Supplementary Figure S3 (data set published in Tomlins et al, 2007). The present study delineates the function of transcription factor EGR3 in prostate cancer.

Early Growth Response 3 was not induced by TPA or serum in prostate cancer cells, contrary to family member EGR1 that also plays a role in prostate cancer (reviewed in Gitenay and Baron, 2009) and is strongly induced by mitogens and growth factors. Both EGR1 and EGR3 share homologous DNA-binding domains 
A
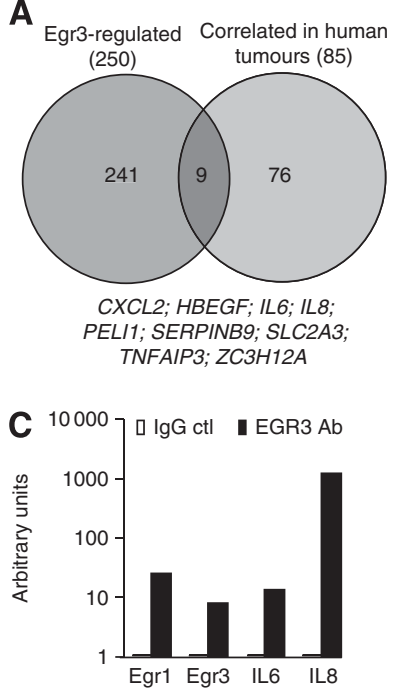

Figure 4. Early Growth Response 3 regulates 116 and IL8 in M12 prostate cancer cells. (A) A Venn diagram compares the list of genes that were induced by EGR3 in M12 cells with the list of genes that were highly correlated with EGR3 in clinical human prostate cancer samples. The list of nine genes found at the intersection is shown below the diagram. (B) Confirmation of EGR3-mediated regulation of gene expression. Total RNA was extracted from stable cell lines control (shSCR-M12) and shEGR3 (shEGR3-cl2 and shEGR3-cl3). The mRNA expression was analysed by quantitative RT-PCR using specific primers. The housekeeping gene GAPDH was used as reference. Expression values obtained for each shEGR3 clone were compared with shSCR control using the $2^{-\Delta \Delta} \mathrm{CT}$ method. (C) Chromatin immunoprecipitation of EGR3 target genes IL6 and IL8. Cells fixed with paraformaldehyde and a specific EGR3 antibody was used to capture protein-DNA complexes. Nonimmune IgG was used as negative control. Promoter regions were amplified by real-time $\mathrm{QPCR}$ and analysed by electrophoresis on agarose gels.

and bind essentially to the same promoter sequences (Swirnoff and Milbrandt, 1995). However, our observation that the two transcription factors are activated by distinct stimuli suggests that they play distinct biological roles.

It was observed that the conditioned medium from M12 cells had a paracrine effect on low-expressing P69 cells, suggesting that high levels of EGR3 in M12 cells may be caused by an autocrine effect. The cytokine or growth factor that is secreted by M12 cells and can induce EGR3 has not been identified yet. As shown in our study, EGR3 increases the expression of various cytokines and other secreted proteins and therefore could regulate its own inducer. It will be interesting, following identification of this (or these) activator (s), to examine whether its production by prostate epithelial cells or by the environment is common in human prostate cancer and correlates with EGR3 expression.

We demonstrate that EGR3 regulates the expression of $\sim 100$ genes involved in inflammation. Indeed, the list of genes regulated by EGR3 was very highly enriched in genes with function in defense, immune response, and inflammation. Many of these genes map to canonical inflammatory pathways such as the NF- $\kappa \mathrm{B}$ pathway and to signalling pathways activated by IL6 and IL1.

Interestingly, two prominent targets of EGR3 in prostate cancer cells were cytokines IL6 and IL8, and they are of particular interest because of the crucial role that these pro-inflammatory cytokines play in prostate cancer progression. The expression of IL6 and IL8 is increased in localised prostate cancer compared with normal prostate and both cytokines have well-known roles in the initiation and progression of prostate cancer (Waugh and Wilson, 2008;
A

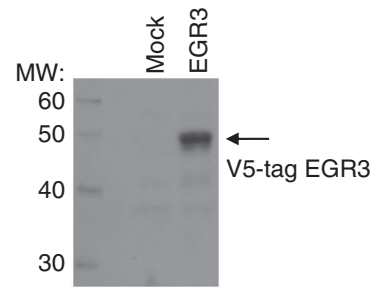

B
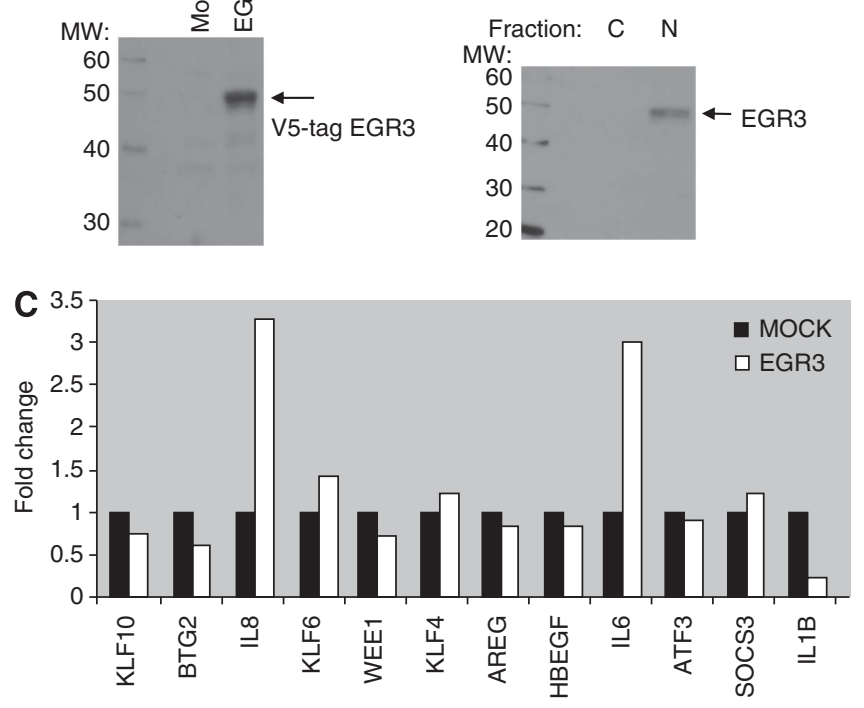

Figure 5. Forced expression of EGR3 induces IL6 and IL8 in P69 cells. (A) Early Growth Response 3 transfection in P69 cells. Cells were transfected with a tag-EGR3 expression plasmid or an empty plasmid as described in the Materials and Methods. Transfected cells were lysed and EGR3 protein expression was analysed by western blotting using antibodies against the V5 tag. (B) Subcellular localisation of EGR3 in transfected P69 cells. Untreated cells were lysed and fractionated using a commercial kit, followed by western blotting. (C) Total RNA was extracted from control or EGR3-expressing P69 cells and the mRNA expression of various EGR3-correlated genes was analysed by real-time qPCR. The housekeeping gene GAPDH was used as reference.

Expression values were compared using the $2^{-\Delta \Delta} \mathrm{CT}$ method.

Culig and Puhr, 2012). These cytokines contribute to the emergence of castration-resistant prostate cancer through regulation of androgen receptor signalling (Waugh and Wilson, 2008; Culig and Puhr, 2012). The expression of both IL6 and IL8 is associated with angiogenesis, tumour cell motility and metastasis, and immune cells chemotaxis. They also act as autocrine growth factors that increase the proliferation of normal prostate epithelial cells and prostate tumour cells (Giri et al, 2001). In addition, IL6 promotes an epithelial-mesenchymal transition that may contribute to metastasis (Rojas et al, 2011). The expression of IL6 and that of IL8 is similarly increased through activation of activating protein 1 (AP1) and NF- $\kappa \mathrm{B}$ transcription factor signalling in response to inflammatory signals and in response to various stresses (Waugh and Wilson, 2008; Taniguchi and Karin, 2014). This study identifies EGR3 as a new regulator of IL6 and IL8 expression in human prostate cancer. Thus, both cytokines were decreased upon EGR3 silencing in M12 prostate cancer cells, and were increased in P69 cells transfected with EGR3 compared with control cells. The IL6 and IL8 promoters do contain EGR-binding sites, and EGR3 was bound to both IL6 and IL8 promoters in a chromatin immunoprecipitation assay.

The clinical relevance of EGR3 function in prostate cancer comes from microarray-based evidence that EGR3 expression in human clinical samples highly correlates with IL6 and IL8 expression (Pio et al, 2013). Future studies may also look at the coexpression of EGR3, IL6, and IL8 at the protein level in human tumour tissue. Alternatively, animal models may help unravel the in vivo relationship between ERG3 and its regulated cytokines. As EGR3 is often overexpressed in human prostate tumours compared with normal tissue, we propose that it may contribute to the state of chronic inflammation that is frequently seen in prostate cancer. Our study also suggests that EGR3 causes excessive production of 
cytokines IL6 and IL8 in some tumours and therefore may directly contribute to disease progression.

Overall, this study identifies a new player in prostate cancer and points to EGR3 as a potential therapeutic target or biomarker for prostate cancer.

\section{ACKNOWLEDGEMENTS}

This work was supported by the National Institutes of Health Strategic Partnering to Evaluate Cancer Signatures (SPECS) Consortium Grant U01 CA1148102, the National Cancer Institute Early Detection Research Network (EDRN) Consortium Grant U01 CA152738, the University of California of Irvine Faculty Career Development Award (to Dr Z Jia), and a gift from the California Women's Auxiliary of the Veterans of Foreign Wars.

\section{CONFLICT OF INTEREST}

D Mercola is a stockholder of Proveri Inc. of San Diego, a biotechnology start-up company developing prostate cancerrelated tests. VT Baron is a consultant for PellFiCure Pharmaceuticals, Inc., a company that develops combination therapies for prostate cancer.

\section{REFERENCES}

Bae VL, Jackson-Cook CK, Maygarden SJ, Plymate SR, Chen J, Ware JL (1998) Metastatic sublines of an SV40 large T antigen immortalized human prostate epithelial cell line. Prostate 34: 275-282.

Balkwill F, Charles KA, Mantovani A (2005) Smoldering and polarized inflammation in the initiation and promotion of malignant disease. Cancer Cell 7: 211-217.

Balkwill FR, Mantovani A (2012) Cancer-related inflammation: common themes and therapeutic opportunities. Semin Cancer Biol 22: 33-40.

Bardia A, Platz EA, Yegnasubramanian S, De Marzo AM, Nelson WG (2009) Anti-inflammatory drugs, antioxidants, and prostate cancer prevention. Curr Opin Pharmacol 9: 419-426.

Ben-Baruch A (2006) Inflammation-associated immune suppression in cancer: the roles played by cytokines, chemokines and additional mediators. Semin Cancer Biol 16: 38-52.

Brawley OW (2012) Prostate cancer epidemiology in the United States. World J Urol 30: 195-200.

Chan JM, Feraco A, Shuman M, Hernandez-Diaz S (2006) The epidemiology of prostate cancer-with a focus on nonsteroidal anti-inflammatory drugs. Hematol Oncol Clin North Am 20: 797-809.

Culig Z, Puhr M (2012) Interleukin-6: a multifunctional targetable cytokine in human prostate cancer. Mol Cell Endocrinol 360: 52-58.

De Marzo AM, Platz EA, Sutcliffe S, Xu J, Gronberg H, Drake CG, Nakai Y, Isaacs WB, Nelson WG (2007) Inflammation in prostate carcinogenesis. Nat Rev 7: 256-269.

De Nunzio C, Kramer G, Marberger M, Montironi R, Nelson W, Schroder F, Sciarra A, Tubaro A (2011) The controversial relationship between benign prostatic hyperplasia and prostate cancer: the role of inflammation. Eur Urol 60: 106-117.

de Visser KE, Korets LV, Coussens LM (2005) De novo carcinogenesis promoted by chronic inflammation is B lymphocyte dependent. Cancer Cell 7: 411-423.

Giri D, Ozen M, Ittmann M (2001) Interleukin-6 is an autocrine growth factor in human prostate cancer. Am J Pathol 159: 2159-2165.

Gitenay D, Baron VT (2009) Is EGR1 a potential target for prostate cancer therapy? Future Oncol 5: 993-1003.

Gomez-Martin D, Diaz-Zamudio M, Galindo-Campos M, Alcocer-Varela J (2010) Early growth response transcription factors and the modulation of immune response: implications towards autoimmunity. Autoimmun Rev 9: 454-458.

Grivennikov SI, Greten FR, Karin M (2010) Immunity, inflammation, and cancer. Cell 140: 883-899.
Gurel B, Lucia MS, Thompson Jr IM, Goodman PJ, Tangen CM, Kristal AR, Parnes HL, Hoque A, Lippman SM, Sutcliffe S, Peskoe SB, Drake CG, Nelson WG, De Marzo AM, Platz EA (2014) Chronic inflammation in benign prostate tissue is associated with high-grade prostate cancer in the placebo arm of the Prostate Cancer Prevention Trial. Cancer Epidemiol Biomarkers Prev 23: 847-856.

Hanahan D, Weinberg RA (2011) Hallmarks of cancer: the next generation. Cell 144: 646-674.

Harris RE (2009) Cyclooxygenase-2 (cox-2) blockade in the chemoprevention of cancers of the colon, breast, prostate, and lung. Inflammopharmacology 17: 55-67.

Huang da W, Sherman BT, Lempicki RA (2009a) Systematic and integrative analysis of large gene lists using DAVID bioinformatics resources. Nat Protoc 4: 44-57.

Huang da W, Sherman BT, Zheng X, Yang J, Imamichi T, Stephens R, Lempicki RA (2009b) Extracting biological meaning from large gene lists with DAVID. Curr Protoc Bioinformatics Chapter 13: Unit 13.11.

Inoue A, Omoto Y, Yamaguchi Y, Kiyama R, Hayashi SI (2004) Transcription factor EGR3 is involved in the estrogen-signaling pathway in breast cancer cells. J Mol Endocrinol 32: 649-661.

Kanterman J, Sade-Feldman M, Baniyash M (2012) New insights into chronic inflammation-induced immunosuppression. Semin Cancer Biol 22: $307-318$.

Karin M (2009) NF-kappaB as a critical link between inflammation and cancer. Cold Spring Harb Perspect Biol 1: a000141.

Kim R, Emi M, Tanabe K (2007) Cancer immunoediting from immune surveillance to immune escape. Immunology 121: 1-14.

Lewis CE, Pollard JW (2006) Distinct role of macrophages in different tumor microenvironments. Cancer Res 66: 605-612.

Liao F, Ji MY, Shen L, Qiu S, Guo XF, Dong WG (2013) Decreased EGR3 expression is related to poor prognosis in patients with gastric cancer. J Mol Histol 44: 463-468.

Lin EY, Pollard JW (2004) Role of infiltrated leucocytes in tumour growth and spread. Br J Cancer 90: 2053-2058.

Mantovani A, Germano G, Marchesi F, Locatelli M, Biswas SK (2012) Cancer-promoting tumor-associated macrophages: new vistas and open questions. Eur J Immunol 41: 2522-2525.

Mittal D, Gubin MM, Schreiber RD, Smyth MJ (2014) New insights into cancer immunoediting and its three component phases-elimination, equilibrium and escape. Curr Opin Immunol 27C: 16-25.

Nozawa H, Chiu C, Hanahan D (2006) Infiltrating neutrophils mediate the initial angiogenic switch in a mouse model of multistage carcinogenesis. Proc Natl Acad Sci USA 103: 12493-12498.

Perez-Cadahia B, Drobic B, Davie JR (2011) Activation and function of immediate-early genes in the nervous system. Biochem Cell Biol 89: 61-73.

Pio R, Jia Z, Baron VT, Mercola D (2013) Early growth response 3 (Egr3) is highly over-expressed in non-relapsing prostate cancer but not in relapsing prostate cancer. PLoS One 8: e54096.

Plymate SR, Tennant M, Birnbaum RS, Thrasher JB, Chatta G, Ware JL (1996) The effect on the insulin-like growth factor system in human prostate epithelial cells of immortalization and transformation by simian virus-40 T antigen. J Clin Endocrinol Metabol 81: 3709-3716.

Pruthi RS, Wallen EM (2005) Cyclooxygenase-2: a therapeutic target for prostate cancer. Clin Genitourin Cancer 4: 203-211.

Rasmussen LM, Frederiksen KS, Din N, Galsgaard E, Christensen L, Berchtold MW, Panina S (2010) Prolactin and oestrogen synergistically regulate gene expression and proliferation of breast cancer cells. Endocr Relat Cancer 17: 809-822.

Rojas A, Liu G, Coleman I, Nelson PS, Zhang M, Dash R, Fisher PB, Plymate SR, Wu JD (2011) IL-6 promotes prostate tumorigenesis and progression through autocrine cross-activation of IGF-IR. Oncogene 30 2345-2355.

Schneider CA, Rasband WS, Eliceiri KW (2012) NIH Image to ImageJ: 25 years of image analysis. Nat Methods 9: 671-675.

Sciarra A, Mariotti G, Salciccia S, Autran Gomez A, Monti S, Toscano V, Di Silverio F (2008) Prostate growth and inflammation. J Steroid Biochem Mol Biol 108: 254-260.

Stock D, Groome PA, Siemens DR (2008) Inflammation and prostate cancer: a future target for prevention and therapy? Urol Clin North Am 35: 117-130vii. Suzuki T, Inoue A, Miki Y, Moriya T, Akahira J, Ishida T, Hirakawa H, Yamaguchi Y, Hayashi S, Sasano H (2007) Early growth responsive gene 
3 in human breast carcinoma: a regulator of estrogen-meditated invasion and a potent prognostic factor. Endocr Relat Cancer 14: 279-292.

Swirnoff AH, Milbrandt J (1995) DNA-binding specificity of NGFI-A and related zinc finger transcription factors. Mol Cell Biol 15: 2275-2287.

Taniguchi K, Karin M (2014) IL-6 and related cytokines as the critical lynchpins between inflammation and cancer. Semin Immunol 26: 54-74.

Tomlins SA, Mehra R, Rhodes DR, Cao X, Wang L, Dhanasekaran SM, Kalyana-Sundaram S, Wei JT, Rubin MA, Pienta KJ, Shah RB, Chinnaiyan AM (2007) Integrative molecular concept modeling of prostate cancer progression. Nat Genet 39: 41-51.

Wang XD, Reeves K, Luo FR, Xu LA, Lee F, Clark E, Huang F (2007) Identification of candidate predictive and surrogate molecular markers for dasatinib in prostate cancer: rationale for patient selection and efficacy monitoring. Genome Biol 8: R255.

Wang Y, Xia XQ, Jia Z, Sawyers A, Yao H, Wang-Rodriquez J, Mercola D, McClelland M (2010) In silico estimates of tissue components in surgical samples based on expression profiling data. Cancer Res 70: 6448 .
Waugh DJ, Wilson C (2008) The interleukin-8 pathway in cancer. Clin Cancer Res 14: 6735-6741.

Welch DR, Schissel DJ, Howrey RP, Aeed PA (1989) Tumor-elicited polymorphonuclear cells, in contrast to "normal" circulating polymorphonuclear cells, stimulate invasive and metastatic potentials of rat mammary adenocarcinoma cells. Proc Natl Acad Sci USA 86: 5859-5863.

Wieland GD, Nehmann N, Muller D, Eibel H, Siebenlist U, Suhnel J, Zipfel PF, Skerka C (2005) Early growth response proteins EGR-4 and EGR-3 interact with immune inflammatory mediators NF-kappaB p50 and p65. J Cell Sci 118: 3203-3212.

This work is published under the standard license to publish agreement. After 12 months the work will become freely available and the license terms will switch to a Creative Commons AttributionNonCommercial-Share Alike 4.0 Unported License.

Supplementary Information accompanies this paper on British Journal of Cancer website (http://www.nature.com/bjc) 